(Received 7 October, 1998 ; Accepted 1 December 1998)

\title{
In-Plane Distribution of Paper Absorbency Measured by Liquid Absorption Profilometer
}

\author{
Toshiharu Enomae ${ }^{* 1}$, Hiroshi Kataoka ${ }^{* 2}$ and Fumihiko Onabe*1 \\ ${ }^{* 1}$ The University of Tokyo, 1-1-1, Yayoi, Bunkyo-ku, Tokyo 113-8657 \\ *2ISI-Dentsu of America, Inc., 1114 Avenue of Americas, New York, NY10036, USA
}

\begin{abstract}
A novel apparatus for quantitative evaluation of a liquid, particularly, water absorption profile of paper was developed. The underlying principle for this method is similar to that of Bristow's method. This system is characterized by an applicator head with a small opening scanning a paper surface and by the detection system using an optical sensor pursuing the meniscus of water filled in a glass capillary connected with the applicator head. The level of sheet formation evidently affected the variation of water absorption rate profile, but the effect was observed only when the contact time was sufficiently long. The coefficient of variation for the worse formation was $36 \%$ higher than that for the better formation at a contact time of $3 \mathrm{~s}$, while it was almost the same at a contact time of $0.2 \mathrm{~s}$. However, no distinct correlation such as accordance of peaks was found between the transmitted light profile and the absorption rate profile. The density variation inducing pore size distribution near the surface layer may affect the absorption rate variation. Additionally, the influence of sizing degree was discussed. This novel system might be applied to clarify effects of water absorbency of basepaper on coating and adequate ink absorption in inkjet printing.
\end{abstract}

\section{Introduction}

Recently mottling has been one of the significant problems associated with print quality of coated papers. The term "mottling" is generally defined as in-plane visual unevenness of ink density. This phenomenon occurs not only to coated grades, but also to uncoated ones. Uncoated paper with rough surfaces results in uneven distribution of ink density in an excessive calendering operation that forms dense and bulky regions. Regarding coated paper, the microscopic structure of coatings is supposed to govern unevenness of ink absorption.

It has been pointed out that binder migration is one of the mechanism associated with occurrence of mottling. Binder migration is the phenomenon where binders, such as starch and latex, migrate towards the surface of coating layer during drying. However, Arai [1] reported that coated paper with high latex concentration at the surface does not always cause mottling. This explains that binder migration does not directly cause it, but that unevenness of surface latex concentration tends to cause mottling as shown by Eklund[2], Norrdahl[3] and Whalen-Shaw [4].

In-plane unevenness of coat weight has also been pointed out as one of the causes for mottling. Engström [5] reported that the binder distribution correlated with coat weight distribution, and this correlation depended on the basepaper and on the coating color. Saito et al. [6] showed that the Croda ink density correlated much better with coat weight distribution than with the surface latex concentration. Kohno[7] found that the basis weight (not coat weight) profile of coated paper, measured with $\beta$-ray, correlated well with the ink density profile and suggested that the printing pressure variation was its cause. So, this should be another factor as well as coat weight and surface latex concentration. 
Coat thickness distribution has been also pointed out as a cause for mottling. Kent [8] examined the coat thickness variations using a mathematical analysis based on Walsh functions. He concluded that high coat thickness areas were mottled areas with less ink absorption because, he speculated, (a) it took longer time to dry in those areas, (b) migrated latex filmed and sealed pores of the surface, and (c) this prevented rapid ink absorption.

All the morphological parameters of coated paper described above, such as distribution of latex surface concentration, coat weight and coat thickness, are supposed to originate from unevenness of the basepaper in various ways. Concerning latex surface concentration, if basepaper has regions of different absorbency from place to place, wet coating consolidates quickly and reaches the immobilization point early in absorbent regions. Therefore, those regions have a lower latex concentration near the surface than less absorbent regions. It follows that occurrences of ink mottling indirectly stems from the non-uniform absorbency distribution in the basepaper. More roughening resulting from fiber swelling in absorbent regions of the basepaper is considered to tend to give higher levels of coat weight and thickness. Finally, those morphological non-uniformity should lead to non-uniform ink density.

Internal loading and pre-coating with latex or starch have been performed as a means to make the absorbency of basepaper uniform $[10,11]$. However, the evaluation of these effects was restricted to subjective ratings over trials of coating and printing practice. Although these may be suitable for product management or quality control, the basic mechanisms need to be systematically investigated. Howarth and Schindler[12] reported on the uniformity of the wetting time over the surface of a paper sheet by rising a stained water surface and making it contact with the sheet horizontally held. They measured the area where water oozed over the opposite side of the paper. But this method does not exactly simulate coating processes because transudation of water occurs near the surface layer in actual coating processes. Furthermore, it should be difficult to wet a paper sheet uniformly over the whole surface because water rising through the cylinder, when overflows, is not flat at the surface.
From the standpoint of the development of apparatus, spiral scan type Bristow tester named DSA (Dynamic Scanning Absorptometer) was developed by Kuga et al[13]. In this apparatus, a Bristow wheel lies horizontally and a circular side of the wheel is used as a sample stage instead of the circumference. Just one spiral scan is long enough to obtain data to draw a whole Bristow curve.

In Pulp and Paper Research Institute of Canada, a new apparatus called water absorption tester is under development to investigate inkjet print quality in terms of feathering and bleed through $[14,15]$. The tester has a similar applicator head and a scan mechanism as the absorption profilometer in this study. But, their tester was designed to estimate lateral penetration of printed inkjet dots.

In the present study, we aimed to evaluate the in-plane unevenness of absorption rate of basepaper. The quantitative evaluation achieved by our new technique allowed to compare it between different papers easily and gave a clue to the correlation of ink mottling with basis weight and density distribution of basepaper, which are fundamental and likely to affect the coating distribution[12].

\section{Experimental}

\subsection{Development of Absorption Profilometer}

An original apparatus for absorption profilometry, that is, for measurement of in-plane non-uniformity of liquid transfer rate of paper was developed. The liquid used in this work was water. The principle of measurement is based on that of the Bristow method [16]. Figure 1 shows the schematic diagram of this apparatus. It consists of the sample stage, applicator head, glass capillary (Iwaki Glass Inc. ,Japan) and the meniscus sensor controlled by a personal computer (PC-9801XL, NEC Inc., Japan). A paper sample mounted on the stage moves horizontally at a precisely constant and variable speed.

Figure 2 illustrates an enlargement of the applicator head. It is made of a stainless tube for liquid chromatography having an inside diameter (ID) of 1.0 $\mathrm{mm}$. A tube of $0.5 \mathrm{~mm}$ ID was also tried for a high resolution. A flexible Teflon tube connects the applicator tube with the glass capillary having an ID of $0.5 \mathrm{~mm}$. Water flows continuously from the meniscus 


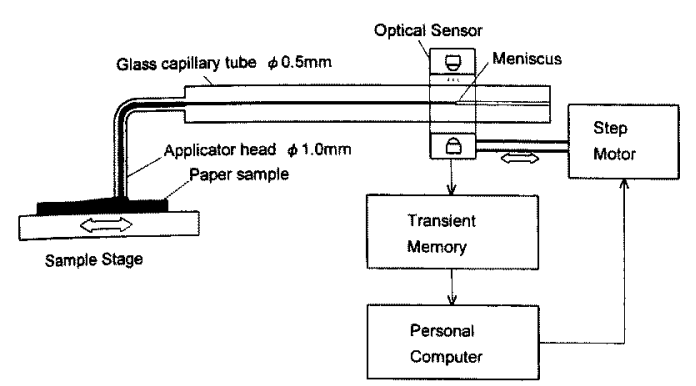

Fig. 1 Schematic diagram of water absorption profilometer.

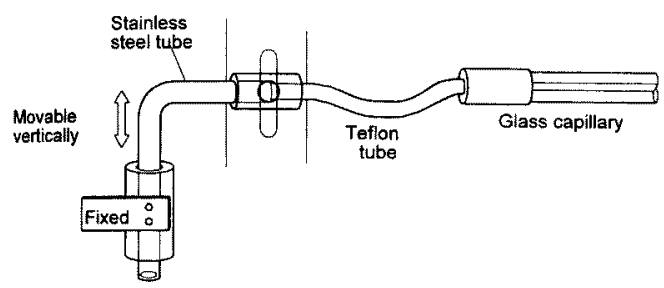

Fig. 2 Schematic diagram of liquid applicator head fixed so that it moves only vertically in scanning.

present in the glass capillary to the tip of the applicator head. Movement of the meniscus in accordance with water transfer to paper is pursued by the optical sensor to detect the meniscus location. The volume of transferred water is calculated from the pursuit distance of the sensor. The sensor is driven by a step motor with a unit step of $0.2 \mathrm{~mm}$, that is, about $0.04 \mu \mathrm{L}$ in water volume. The sensor consists of a light-emitting diode (LED) and a phototransistor (TLN101A and TPS601A, respectively, Toshiba Inc., Japan), both of which works in the infrared range of light, thus are suitable for detecting a water meniscus with high directivity.

The unique and excellent features of this apparatus are as follows: The situation of the contact of the applicator head with a paper surface, hence the way of water transfer is constant along a scan on the surface. Such advantage could not be achieved by the method of wetting the whole surface area at one time. This characteristic, to be exact; cannot be available with the Bristow applicator head, where residual liquid in it decreases with transfer and the transferred volume was slight at the end of the trace. In addition, this method facilitates comparison between different samples because it evaluates absorption unevenness quantitatively.

The tip of the applicator head was polished carefully not to scratch the paper surface. The external force applied to the applicator head to press the paper surface was $0.12 \mathrm{~N}$ and then the pressure was about $0.15 \mathrm{MPa}$, which is close to the pressure prescribed in Bristow's method, i.e. $0.1 \mathrm{MPa}$.

To adjust the pressure of water at a wetting point, the effect of interfacial tension at the meniscus in the glass capillary is not negligible because of its very narrow passage. Therefore, the glass capillary was held about $50 \mathrm{~mm}$ above the applicator head so that no external pressure would be developed to water at a wetting point.

\subsection{Formation Measurement}

If a local basis weight distribution is assumed to be sheet formation, the formation is conventionally evaluated using several kinds of radiography like $\beta$-ray and soft X-ray. For safety and simplicity, visible light was used in this study to determine the formation level although it has some inaccuracies[17-19] to identify the transmitted light distribution with the basis weight distribution. However, for uncalendered handsheets without filler, light transmittance is approximately a function of basis weight. Enomae and Kuga[20] found that the pressure in wet-pressing hardly affected the light transmittance. This implies that, even if there happens non-uniform wet-pressing over a handsheet, the non-uniform density distribution caused by it hardly affects the non-uniformity of the transmitted light image. So, "formation profile" referred-to here means the profile of transmitted light intensity, that is, gray level in its images.

A paper sample was placed on the frosted glass through which a fluorescent lamp illuminated the sample. The frosted glass served to scatter light and homogenize the illumination. An anti-reflective transparent glass was placed on the sample. Transmitted light images were taken with a $C C D$ video camera (TI-22A, NEC Inc., Japan) and the dynamic range of input signal was adjusted in an image analyzer (LA-500, PIAS corp., Japan) so that the difference between a maximum and a minimum of the transmitted light intensity (gray level) would be expanded and optimized. The actual size of $1 \mathrm{~mm} \times 1 \mathrm{~mm}$ on the paper 
sample was equivalent to 6 pixels $\times 6$ pixels in the image. The transmitted light images of the sample papers were saved ahead of the subsequent water absorption test. After the water absorption test, the scanned location was sought from the saved image by visual comparison. Then, the corresponding line profile of gray level was extracted from the whole image in such a way that the mean gray was calculated sequentially along the scan line from adjacent 5 pixels in the cross-scan direction, equivalent to $0.8 \mathrm{~mm}$ on the actual paper sample.

\subsection{Materials and Sample Preparation}

Commercially available woodfree paper, a copy paper, was mainly used. The basis weight and the Stökigt sizing degree were $64 \mathrm{~g} / \mathrm{m}^{2}$ and $34 \mathrm{~s}$, respectively. Machine-made woodfree papers with different sizing degrees were also used to examine the effect of sizing degree on the absorption curve. The sizing degree was varied by adding the different amount of alkyl ketene dimer (AKD) to the furnish. The Stökigt sizing degree was about $0,<1$, and $48.6 \mathrm{~s}$ for weakly-, intermediately-, and hard-sized paper, respectively. The basis weight of the three samples was about $75 \mathrm{~g} / \mathrm{m}^{2}$. Scans were made by the absorption profilometer along the machine direction to reduce the risk of elongation during a scan.

Handsheets were prepared to examine the effect of sheet formation. The furnish consisted of hardwood bleached kraft pulp beaten for 6000 revolutions in PFI mill. A $1.3 \mathrm{wt}-\%$ rosin size to that of pulp and aluminum sulfate to adjust $\mathrm{pH}$ to 4.5 were added. Sheets of two different levels of formation were prepared according to JIS in most procedures. Sheets of the worse formation were prepared in the following modified manner. The pulp slurry was allowed to stand for 5 minutes with the slurry surface level at $100 \mathrm{~mm}$ (normally $350 \mathrm{~mm}$ ) above the wire, then drained. Every sheet was dried according to the ring-restraint drying method. The Stökigt sizing degree was about $25 \mathrm{~s}$.

\section{Results and Discussion}

\subsection{Effect of Scanning Speed on Water Transfer}

Figure 3 shows water transfer behavior basically obtained using the absorption profilometer at different scanning speed of the applicator head. The horizontal axis is the scan distance of the applicator head on the paper surface. The vertical axis is the transferred

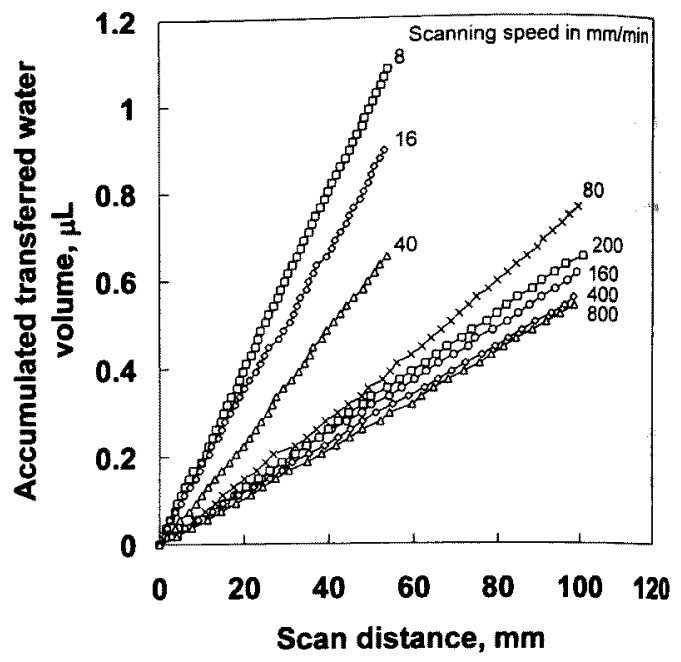

Fig. 3 Transferred water volume accumulated with scan distance at various constant scanning speeds.

water volume accumulated with the scan. Every relationship was, in average, linear regardless of the scanning speed. At higher scanning speeds, the slope of the curves came close while a small difference in the scanning speed made a lot of differences in the slope at lower scanning speeds.

The final goal of this work is to determine the degree of variation in absorption rate from point to point. The level of linearity of the relationship found in Fig. 3 represents the degree of non-uniformity of paper absorbency. In other words, the higher the level of linearity, the more uniform the in-plane paper absorbency. More discussion regarding unevenness of paper will be given later. First of all, the data from this new technique were compared with that of Bristow's method to confirm that this profilometer provides reliable results.

\subsection{Comparison with Bristow's Method}

When only the average slope of the curves shown in Fig. 3 is taken into account, all the slopes together at each contact time provides a Bristow's curve. The contact time was calculated by dividing the inside diameter, that is, $1 \mathrm{~mm}$ by a scanning speed. However, the contact time, strictly speaking, is varied along the $1 \mathrm{~mm}$ diameter perpendicular to the scan direction because the opening is circular. In this sense, the precise contact time is difficult to calculate. However, this 
deviation was actually ignored because the longest contact time located in the center of the opening was assumed to govern the practical contact time. This assumption was found to be allowed as mentioned below.

Figure 4 shows a water transfer curve determined by the conventional Bristow's method and by the absorption profilometry. The fact that the data points by the two methods are well plotted on the identical curve suggest that the contact time could be calculated with no problem by assuming that the longest contact time governed the mean contact time. Another problem inherent to the absorption profilometry is that the narrow passage of glass capillary might cause viscous drag in water flow. However, the comparison of the present method with the conventional Bristow's method shows that the values measured by this method was sufficiently adequate even at the highest scan speed. Consequently, the reliability of the absorption profilometry was empirically guaranteed.

\subsection{Effect of Sizing Degree on Water Transfer Curve}

The relationship between the transferred water volume and the scan distance shown in Fig. 3 is affected by sheet properties such as sizing degree, surface

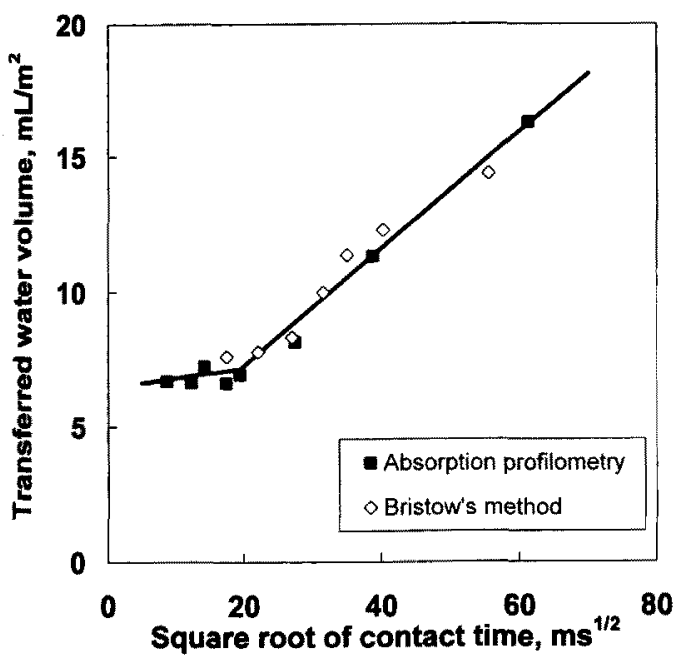

Fig. 4 Water transfer curve measured by Bristow's method and by absorption profilometry. Inside diameter of applicator was $1.0 \mathrm{~mm}$. roughness, basis weight, and density. The factors of the profilometer such as scanning speed and inside diameter of the applicator head are also supposed to affect this relationship.

Figure 5 shows the changes in accumulated transferred water volume with scan distance at a constant scanning speed $(200 \mathrm{~mm} / \mathrm{min})$ for commercial woodfree papers of three different sizing degrees. It was observed that the mean transfer rate, that is, the slope of the curves decreased with the sizing degree.

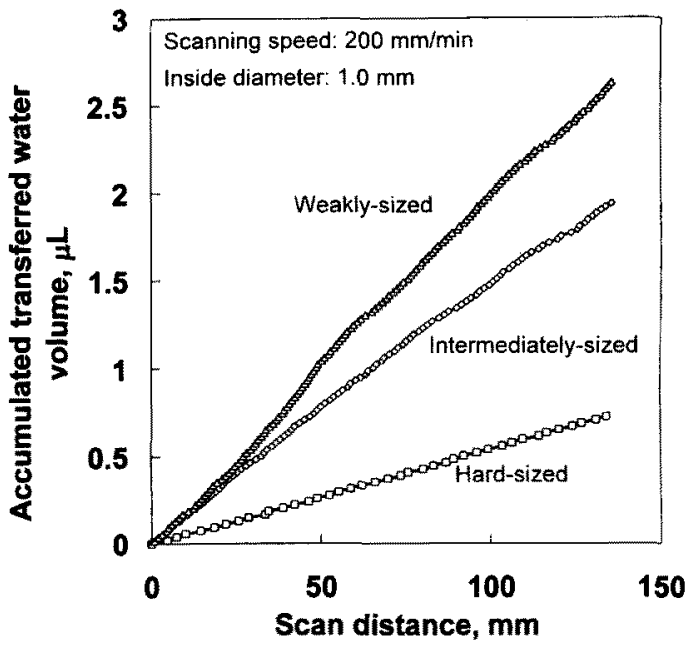

Fig. 5 Water transferred volume accumulated with scan length at a constant scanning speed for variously sized papers. Deviation from linearity indicates in-plane unevenness of paper absorbency.

\subsection{Definition of Absorption Profile}

Curves of accumulated transferred water volume versus scan distance as plotted in Figs 3 and 5 can be converted into an absorption profile. Figure 6 illustrates schematically an example of the absorption profile obtained by differentiating the transferred water volume with regard to the scan distance. The rate of water absorption at position A corresponds to that $\left(V_{a}\right)$ of the inset Bristow's curve representing the absorbency around position A. In the inset Bristow's curve, contact time $T$ is determined by the scanning speed and the inside diameter of the applicator. The absorbency around position $B$ is assumed like the other inset Bristow's curve and $V_{b}$ is plotted in the absorption 


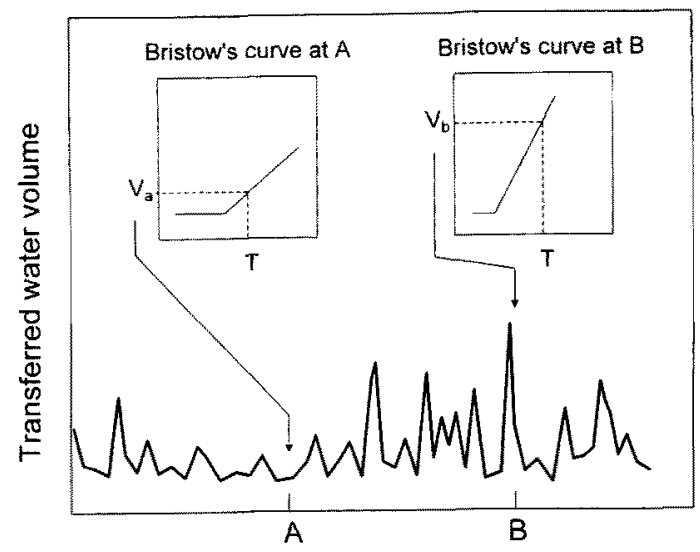

Relative position, $\mathrm{mm}$

Fig. 6 Schematic absorption profile at contact time T. $V_{a}$ and $V_{b}$ are transferred water volumes at position $A$ and $B$, respectively. Inset Bristow's curves represent absorption properties at specified positions.

profile. The shapes of Bristow's curve in the insets are practically unknown except values $V_{a}$ and $V_{b}$.

\subsection{Effect of Sheet Formation on Absorption Profile}

Figure 7 shows an absorption profile (solid line) of a handsheet of the better formation. The formation profile (the profile of transmitted light intensity) just on the same location was also shown (dotted line). Figure 8 shows the results of the same experiment for a handsheet of the worse formation. A comparison between Figs. 7 and 8 exhibited that the worse formation leads to a larger variation as found in the absorption profile. However, the distinct correlation such as accordance of peaks between them was not found even with the sample of the worse formation. Although the formation profile was supposed to influence the absorption profile considerably, this result did not give its enough evidence. Otherwise, the density distribution especially close to the surface may have possibly affected the absorption profile considering that it governs the pore size distribution closely related to the absorption rate. Besides, distributions of fines and size are generally potential to affect it, but those effects are less likely involved in the data for the handsheets. Anyway, the absorption profilometer clearly showed an influence of basis

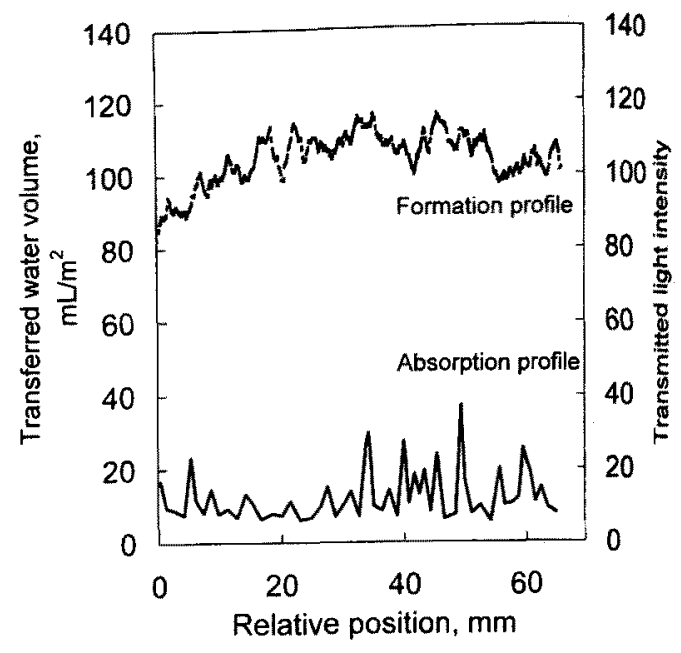

Fig. 7 Comparison between water absorption profile and basis weight profile for a handsheet of the better formation. Scanning speed was $40 \mathrm{~mm} /$ min. Inside diameter was $1.0 \mathrm{~mm}$.

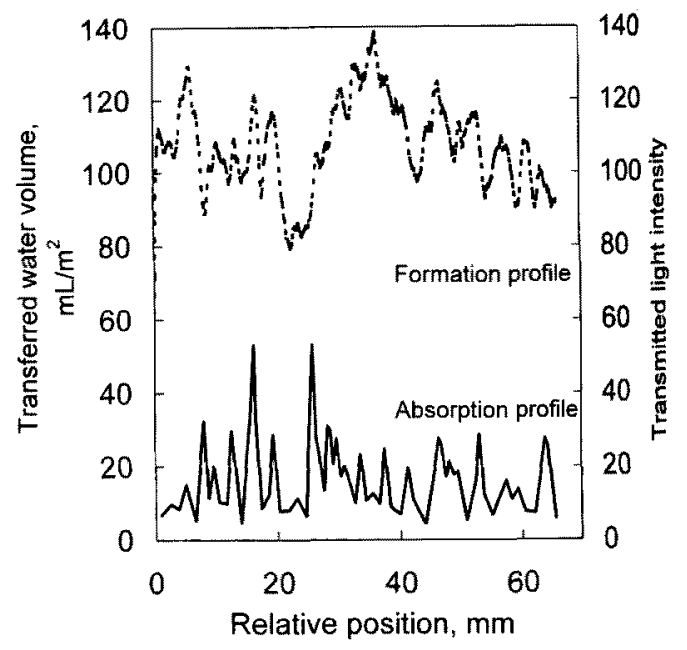

Fig. 8 Comparison between water absorption profile and basis weight profile for a handsheet of the worse formation. Scanning speed was $40 \mathrm{~mm} /$ min. Inside diameter was $1.0 \mathrm{~mm}$.

weight variation on the absorption rate.

Figure 9 shows absorption profiles at different scanning speeds for the two levels of formation. Obviously, at least the extent of variation of the absorption profiles was affected by the formation. Therefore, it follows that the basis weight had some 
influence on the absorption rate profiles. Table 1 shows the statistical values of the water absorption rate at different scanning speeds for the handsheets of the different formation. There was little difference among the coefficient of variation between the two levels of formation at a scanning speed of $300 \mathrm{~mm} / \mathrm{min}$. On the contrary, significant differences were observed at 20 $\mathrm{mm} / \mathrm{min}$. The contact time was 0.2 and $3 \mathrm{~s}$ at 300 and $20 \mathrm{~mm} / \mathrm{min}$, respectively. From Bristow's theory or in view of wetting delay, it was considered that penetration of water had not yet occurred for the initial period of $0.2 \mathrm{~s}$. Therefore, the variation of the profiles at $300 \mathrm{~mm} / \mathrm{min}$ depended, probably, only on the surface roughness variation. The difference in this variation between the two levels of formation was presumably too small to be detected. On the other hand, water had already started to penetrate before $3 \mathrm{~s}$ passed. In summary, the variation of penetration rate was

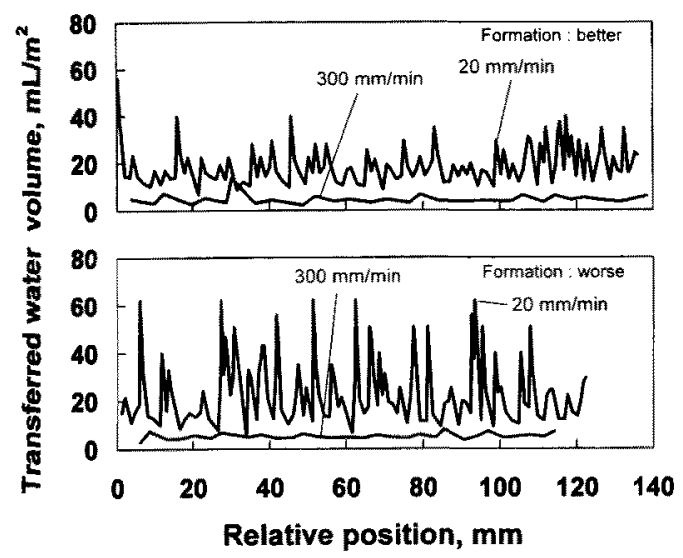

Fig. 9 Comparison of absorption profile variation between papers of better and worse formations at a scanning speed of 20 or $300 \mathrm{~mm} / \mathrm{min}$.

Table 1 Statistical values of water transferred volume for papers of the better and worse formations.

\begin{tabular}{llllll}
\hline Scanning speed, $\mathrm{mm} / \mathrm{min}$ & \multicolumn{2}{c}{300} & \multicolumn{2}{c}{20} \\
\cline { 2 - 6 } Formation & Mean & 5.03 & 5.69 & 19.68 & 25.65 \\
\hline \multirow{2}{*}{$\begin{array}{l}\text { Water transferred } \\
\text { volume, } \mathrm{mL} / \mathrm{m}^{2}\end{array}$} & $\begin{array}{l}\text { Standard } \\
\text { deviation } \\
\text { Coefficient of }\end{array}$ & 2.49 & 2.75 & 8.28 & 14.64 \\
& $\begin{array}{l}\text { variation } \\
\text { varse }\end{array}$ & & 0.48 & 0.42 & 0.57 \\
\hline
\end{tabular}

considered to be influenced by the variation of basis weight as mentioned above, but details of the mechanism should be investigated in further researches.

\section{Conclusions}

A novel technique was developed for measuring an in-plane profile of liquid transfer volume by assembling a prototype applicator head with a small opening and an optical sensor pursuing the meniscus. As a result of the data obtained using this water absorption profilometer, the in-plane variation of an absorption profile of a handsheet was influenced by the basis weight variation at a sufficiently longer contact time. The coefficient of variation for the worse formation was $36 \%$ higher than that for the better formation at a contact time of $3 \mathrm{~s}$, while it was almost the same at a contact time of $0.2 \mathrm{~s}$. However, no distinct correlation was found between the absorption profile and the profile of transmitted light intensity that represents approximately the basis weight variation. This suggests that not only the basis weight variation but also the density variation of paper affected the absorption profile. The novel technique developed in this study could give a clue to solve ink mottling particularly if the lateral scanning is combined to expand the scan range to three dimensional mapping. Additionally, it is expandable to ink absorption behavior in inkjet printing.

This work was partly presented in 1993 TAPPI Advanced Coating Fundamentals Symposium, Minneapolis, MN, USA

\section{References}

1. T. Arai, T., Yamasaki, K. Suzuki, T. Ogura and Y. Sakai, 1988 TAPPI Coating Conference Proceedings, 187, (1988).

2. D. E. Eklund and J. A. Palsanen, Tappi, 53 (10), 1925 (1970).

3. P. C. Norrdahl, 1991 TAPPI Coating Conference Proceedings, 417 (1991).

4. M. Whalen-Shaw and T. Eby, Tappi, 74(12), 188 (1991).

5. G. Engström, M. Rigdahl, J. Kline and J. Anlroos, 1991 TAPPI Coating Conference Proceedings, 169 
(1991).

6. Y. Saito, H. Matsubayashi, Y. Takagishi, K. Miyamoto, and Y. Kataoka, Pan-Pacific Pulp \& Paper Technology Conference Proceedings, 45 (1992).

7. M. Kohno, and T. Hamada, Kamipa Gikyoshi, 40 (3), 295 (1986)

8. H. J. Kent, N. A. Climpson, L. Coggon, J. J. Hopper and P. A. C. Gane, Tappi, 69(5), 78(1986).

9. P. Howarth, and M. K. Schindler, "The aerial distribution of liquid penetration of paper", F. R. C. at Oxford, England, 483-496 (1985).

10. P. Lepoutre, W. Bichard and J. Skowronski, Tappi, 69(12), 66(1986).

11. R. W. Dahlquist, L. J. Welch and R. E. Hardy, Tappi, 73(12), $151(1990)$.

12. H. Kataoka, T. Enomae, F. Onabe and M. Usuda,
Sen'i Gakkai Preprints 1992(S), Tokyo, S-228 (1992).

13. S. Kuga, H. Kataoka, T. Enomae, F. Onabe, N. Yagi and O. Shinkai, Kamipa Gikyoshi, 48(5), 730 (1994).

14. M. Inoue, Private communication (1998).

15. Y.-G. Tsai and M. Sakamoto, 1997 Japan Tappi Annual Meeting Proceedings, 261 (1997).

16. J. A. Bristow, Svensk Papperstid., 70 (19), 623(1967).

17. H. Tomimasu, K. Suzuki, T. Ogura and P. Luner, Tappi, 73(5), $179(1990)$.

18. M. A. MacGregor and P-A Johansson, Tappi, 73 (12), 161(1990).

19. M. A. MacGregor and P-A Johansson, Tappi, 74(1), 187 (1991).

20. T. Enomae and S. Kuga, J. Wood Science, submitted for publication (1999). 